\title{
Degree of optical polarization as a tool for detecting melanoma: proof of principle
}

Daniel C. Louie

Jamie Phillips

Lioudmila Tchvialeva

Sunil Kalia

Harvey Lui

Wei Wang

Tim K. Lee 


\title{
Degree of optical polarization as a tool for detecting melanoma: proof of principle
}

\author{
Daniel C. Louie, ${ }^{a, b}$ Jamie Phillips, ${ }^{b}$ Lioudmila Tchvialeva, ${ }^{b}$ Sunil Kalia, ${ }^{b}$ Harvey Lui, ${ }^{b, c, d}$ Wei Wang, ${ }^{e}$ and \\ Tim K. Lee $e^{a, b, c, d, *}$ \\ aUniversity of British Columbia, School of Biomedical Engineering, Vancouver, Canada \\ bUniversity of British Columbia and Vancouver Coastal Health Research Institute, Department of Dermatology and Skin Science, \\ Vancouver, Canada \\ ${ }^{c}$ British Columbia Cancer Agency, Department of Cancer Control Research, Vancouver, Canada \\ ${ }^{\mathrm{d} B r i t i s h}$ Columbia Cancer Agency, Department of Integrative Oncology, Vancouver, Canada \\ ${ }^{\mathrm{e} H e r i o t-W a t t}$ University, School of Engineering and Physical Sciences, Edinburgh, United Kingdom
}

\begin{abstract}
Determining the optical polarization properties of a skin lesion is a proposed method to differentiate melanoma from other skin lesions. We developed an in vivo Stokes polarimetry probe that fires a laser of known polarization at the skin and measures the Stokes parameters of the backscattered light in one shot. From these measured Stokes parameters, we can calculate the degree of polarization (DOP). Through testing on rough skin phantoms, a correlation between backscattered DOP and skin roughness was identified for both linear and circular input polarization, the latter of which was found to be more useful. In a pilot clinical trial of 69 skin lesions in vivo, it was found that the mean DOP for melanoma (linear input on melanoma: $0.46 \pm 0.09$ ) was greater than that of other lesions (linear input on all other lesions: $0.28 \pm 0.01$ ). This separation is greater for circular polarized input light, and it is likely that circular polarized light's greater sensitivity to surface roughness contributes to this result. In addition, all skin lesions demonstrated a stronger depolarizing effect on circular polarized light than linear polarized light. We have identified DOP as a potentially useful measurement to identify melanoma among other types of skin lesions. () The Authors. Published by SPIE under a Creative Commons Attribution 3.0 Unported License. Distribution or reproduction of this work in whole or in part requires full attribution of the original publication, including its DOI. [DOI: 10.1117/1.JBO.23.12.125004]
\end{abstract}

Keywords: degree of polarization; Stokes vector; roughness; skin cancer; in vivo; skin phantoms.

Paper 180453R received Jul. 16, 2018; accepted for publication Nov. 26, 2018; published online Dec. $15,2018$.

\section{Introduction}

Skin cancer is the most common form of cancer in North America, Australia, and other predominantly fair-skinned populations worldwide, and the incidence of skin cancer has been rapidly increasing over the past several decades. ${ }^{1}$ Skin cancer is typically divided into melanoma and nonmelanoma subcategories. The majority of nonmelanoma skin cancers are keratinocyte carcinomas, namely basal cell carcinoma (BCC) and squamous cell carcinoma (SCC), both of which can often lead to disfigurement and further complications if left untreated. ${ }^{2}$ Malignant melanoma (MM) is associated with a significantly higher mortality rate than keratinocyte carcinoma. There is strong correlation between prognosis and melanoma tumor thickness within the skin, highlighting the importance of early detection and treatment for improving patient outcomes and survival. ${ }^{3}$

Polarimetry has been an increasingly popular method of optical measurement in recent years. In the biomedical field, polarization has been applied to reduce glare in clinical photography, ${ }^{4}$ increase sensitivity to collagen in confocal microscopy, ${ }^{5}$ and measure birefringence in optical coherence tomography. ${ }^{6}$ Of particular interest is direct polarimetry for cancer screening and diagnosis. This has been typically approached with a Mueller matrix model, in which the diagnostic parameters of tissue are determined by the degree to which input polarized light is altered. As a growing field, there is a large variety of

*Address all correspondence to Tim K. Lee, E-mail: tlee@bccrc.ca established and developing instrumentation in the field of tissue polarimetry. ${ }^{7,8}$ Common among polarimetry techniques is the preference for polarization state analyzers and generators that do not require mechanical actuation, which allows for faster in-vivo measurements. This can be achieved using components such as photoelastic modulators ${ }^{9}$ or liquid crystal variable retarders. ${ }^{10}$ The simplest measurement methods include polarimetric probing, ${ }^{11,12}$ which use one-dimensional intensity measurements to calculate polarization properties. Methods become more complex in Mueller matrix imaging, ${ }^{13}$ which requires the acquisition of 16 images, one for each Mueller matrix element. These images are analyzed for specific polarization properties through Lu-Chipman decomposition ${ }^{14}$ or further extended formalisms. ${ }^{15}$

The dominant polarimetric effect in tissue is depolarization, whereby the polarization (DOP) of an optical wave packet becomes randomized due to the stochastic nature of tissue scattering. ${ }^{16}$ The degree of polarization (DOP) is the simplest polarimetric property to quantify because it only requires a Stokes vector for its calculation. DOP is noteworthy as an optical measurement for skin tissue analysis due to the relationship between DOP and surface roughness. Inspired by industrial surface roughness measurement techniques, Tchvialeva et al. ${ }^{17}$ proposed polarization speckle imaging for skin cancer screening. This study found that the speckle contrast and the depolarization ratio of Stokes parameters $S_{1} / S_{0}$, were promising differential features between cancerous and benign lesions due to its correlation to surface roughness. This study measured the mean roughness of a range of potentially suspicious skin lesions and noted that MM and nevus (benign moles) were both 
smoother than other lesion types. However, as roughness was this study's key measurement, it was unable to distinguish similarly smooth nevi from melanomas, which is a crucial task as they often appear visually similar.

In this paper, we demonstrate a proof of principle for using DOP as an in vivo metric to differentiate melanoma from other skin lesions irrespective of surface roughness. We have recently developed a handheld Stokes polarimetry probe that is capable of one-shot, rapid, in vivo DOP measurements with both linear and circular input polarizations. This device is designed to measure a complete Stokes vector while also being portable and cost-effective for clinical adoption. This probe and some of the results of its testing reported here were previously discussed at SPIE Photonics West 2018. ${ }^{18}$ In this paper, the relationship between DOP and surface roughness is demonstrated through the testing of this Stokes polarimetry probe on four different rough skin phantoms. These results show that DOP can differentiate the phantoms based on their optical properties independent of surface roughness. This was followed by a preliminary clinical trial on both cancerous and noncancerous skin lesions in vivo, using the same Stokes polarimetry probe to measure DOP.

\section{Methods and Instruments}

\subsection{Stokes Vector}

Polarization is the orientation of a light wave, which includes linear, elliptical, and circular states. The polarization state of light is formalized using a $4 \times 1$ Stokes vector $\mathbf{S}$ as

$\mathbf{S}=\left[\begin{array}{l}S_{0} \\ S_{1} \\ S_{2} \\ S_{3}\end{array}\right]$

Each of the Stokes vector parameters corresponds to the relationship between two orthogonal states of polarization. Using a Cartesian reference frame, an incident beam of light can be defined in a wave equation in terms of horizontal $X$ and vertical $Y$ axis components, while propagating in the $Z$ direction. $S_{0}$ is the total intensity of light and can be calculated as sum of any two orthogonal components. $S_{1}$ is the difference in intensity between horizontal and vertical linearly polarized components, $S_{2}$ is the difference in intensity between linear polarized components $+45 \mathrm{deg}$ and $-45 \mathrm{deg}$ from the horizontal, and $S_{3}$ is the difference in intensity between right- and left-hand circular polarized components.

The Stokes parameters can be quantitatively described using six different intensity measurements taken with ideal polarization filters: $I_{\mathrm{H}}$ (horizontal, linear), $I_{\mathrm{V}}$ (vertical, linear), $I_{\mathrm{P}}$ (+45 deg, linear), $I_{\mathrm{M}}\left(-45 \mathrm{deg}\right.$, linear), $I_{\mathrm{R}}$ (right-hand circular), and $I_{\mathrm{L}}$ (left-hand circular). These relationships are shown in Eq. (2) which defines the Stokes parameters:

$S_{1}=I_{H}-I_{V}$,

$S_{0}=I_{H}+I_{V}$,

$S_{2}=I_{P}-I_{M}$,

$S_{3}=I_{R}-I_{L}$.

Note that the formalism described here for circular and elliptical polarization is from the perspective of the source. For ease of analysis, the Stokes vector is often normalized such that each Stokes parameter is divided by $S_{0}$. This brings $S_{0}$ to 1 while correspondingly reducing the other parameters.

As demonstrated by Collett, ${ }^{19}$ the Stokes vector can be derived with only four measurements using a single circular polarizer at different rotations. A linear polarizer followed by a quarter-wave plate, with the polarizer's transmission axis at $45 \mathrm{deg}$ from the waveplate's fast axis, can act as a linear polarized filter when receiving light from the polarizer side and a right-hand circular polarized filter when receiving light from the waveplate side. This combined filter can be rotated and flipped successively to act as the above-mentioned $I_{V}, I_{P}, I_{M}$, and $I_{R}$ filters, and from these measurements the complete Stokes vector can be calculated since the sum of any two orthogonal polarization components are equal as in Eq. (3). This relationship and subsequent derivation of the Stokes vector in four parameters are shown in Eq. (4):

$I_{H}+I_{V}=I_{P}+I_{M}=I_{R}+I_{L}$,

$S_{0}=I_{P}+I_{M}$,

$S_{1}=S_{0}-2 I_{V}$,

$S_{2}=I_{P}-I_{M}$,

$S_{3}=2 I_{R}-S_{0}$.

This technique of using a single circular polarizer sequentially can also be applied to using four identical filters and four detectors simultaneously, which underlies the polarization methodology for the probe in this study. The inclusion of four filters eliminates the need for mechanical rotation of a filter and allows for a one-shot measurement as opposed to multiple serial measurements.

We can determine depolarization by calculating the DOP from the Stokes vector, defined through Eq. (5). DOP is a value between $S_{0}$ and 0 , and for a normalized Stokes vector the DOP is a percentage value of uniform polarized content within a given wave packet:

$\mathrm{DOP}=\frac{\sqrt{S_{1}^{2}+S_{2}^{2}+S_{3}^{2}}}{S_{0}}$.

\subsection{Stokes Polarimetry Probe}

Measurements in this study were taken by a handheld Stokes polarimetry probe, the design of which has been previously reported. ${ }^{18,20}$ Briefly, the probe fires a laser diode with known polarization at the skin and measures the backscattered light that returns.

The laser diode has a peak wavelength of $663 \mathrm{~nm}$ (ThorLabs HL6545MG). It is collimated to a diameter of $1 \mathrm{~mm}$, and each measurement is taken through a 1-ms exposure. The incident power on the skin is $63 \mathrm{~mW}$, which is within exposure limitations while also offering a backscattered signal with a detectable intensity. This short exposure also helps to lessen the effect of movement artifacts on the output. For this study, the laser's initial polarization was set to being linear and right-hand circular. Linear polarization was set using a wire-grid linear polarizer (ThorLabs WP12L-VIS) with the transmission axis set to a reference $0 \mathrm{deg}$. Circular polarization was set by adding a quarter-waveplate film (Bolder Vision Optik) after the wire-grid 
polarizer with the fast axis set placed at $45 \mathrm{deg}$ from the reference.

The backscattered light is collected by four photodiodes equipped with film polarizing filters (Bolder Vision Optik) to measure a complete Stokes vector, using the Collett method as described in Sec. 2. The photodiodes (ThorLabs FDS100) have an active area of $3.6 \mathrm{~mm} \times 3.6 \mathrm{~mm}$. They are symmetrically placed radially around the source, and oriented parallel to the target plane under the assumption that the backscattered field is uniform for each photodiode. The measurements of the four photodiodes are averaged over the duration of the laser pulse, and then combined to generate a Stokes vector.

The backscattered light that emerges from the tissue forms a polarization speckle field due to the interference within the backscattered partially coherent light. This field expands conically, ${ }^{21}$ and the photodiode detectors are placed far enough away $(10 \mathrm{~cm})$ to allow for the collection of a uniform speckle field. The detectors measure the spatial average intensity of this speckle pattern, with the Stokes vector and DOP measurements being derived from this average intensity, which is indicative of the bulk polarization behavior. In as of yet unpublished validation testing, the device demonstrated $1.1 \%$ standard error in DOP measurements of ideal polarized light. This is attributed to probe misalignment errors and nonideal circular polarizing film. The probe requires an equal intensity of light to reach each of the photodiodes for accurate polarization measurements. There is also a slight incompatibility between polarizing film intended for monochromatic light and the multispectral profile of a laser diode. These errors were determined to be minor enough to not significantly affect DOP measurements and future iterations of this device will improve upon these errors. In addition, full imaging and spatial analysis of polarization speckle patterns, as opposed to the average measured here, may offer additional information and will be the focus of ongoing study.

The probe head is shown in Fig. 1. The conical front of the probe is placed and sized to ensure that the detectors are fixed $10 \mathrm{~cm}$ away from the target site, and the interior is coated in antireflective velvet. This head is connected to a circuit and a computer for data collection. The software is programmed in a National Instruments LabVIEW system.

\subsection{Skin Phantoms}

The rough color phantoms in this study were constructed as per a previously published recipe. ${ }^{22}$ Roughness was controlled by curing the resin mixture over a metal roughness comparator (Microshurf \#334 comparator, Rubert+Co Ltd., Cheadle, United Kingdom) with seven rough surfaces. The root-mean-square roughness $R q$ values, ranging from 1 to $64 \mu \mathrm{m}$, were within the order of previously reported human skin roughness. ${ }^{23-26}$ The phantom roughness was confirmed by a WYKO NT2000 optical profilometer (Veeco, Tucson, Arizona) with vertical resolution of $0.05 \mu \mathrm{m}$, lateral resolution of $0.5 \mu \mathrm{m}$, and standard error of $10 \%$. Each phantom contains seven zones of different surface roughnesses, of which six were tested by the Stokes polarimetry probe: $2.2,4.5,9,18,34$, and $68 \mu \mathrm{m}$.

The matrix of the phantoms was constructed using SmoothOn brand MoldMax 10T silicone which came as a viscous resin, accompanied by a curative that causes the silicone to cure at room temperature. Silc-pig silicone pigments (Smooth-On, Inc.) were added as absorbing particles. These liquid pigments came in nine colors and create a homogeneous colored solution when mixed with silicone resin; human skin color can be

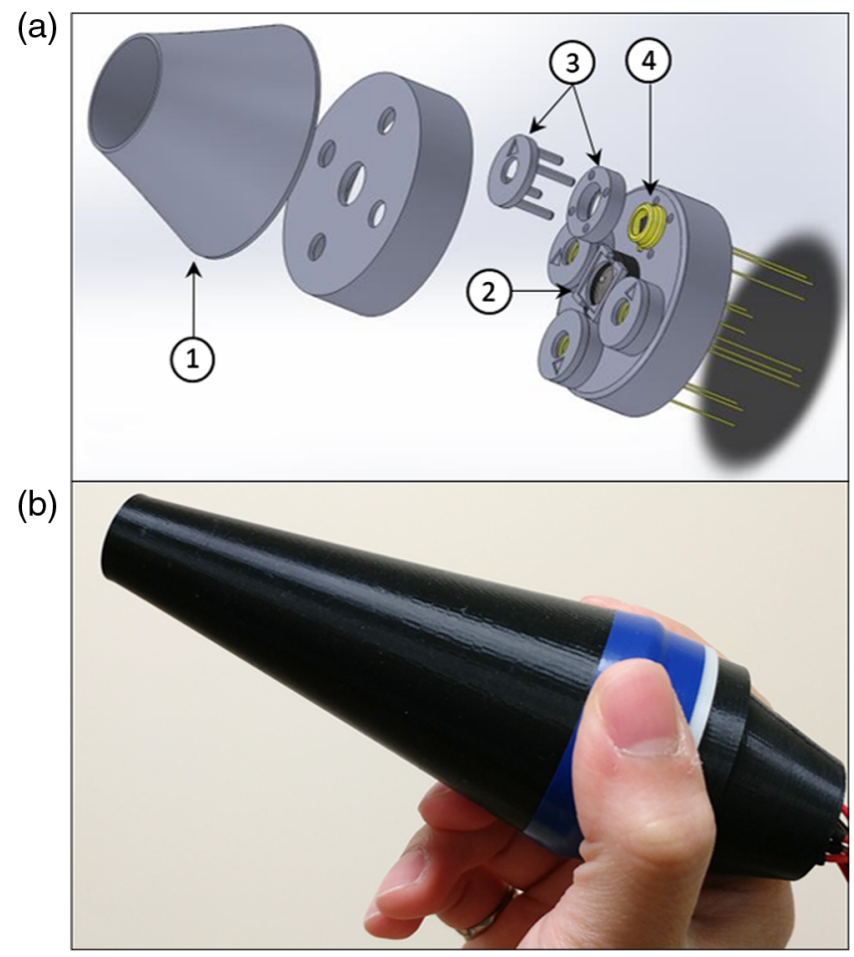

Fig. 1 (a) Illustration of the Stokes polarimetry probe head components, including (1) conical front piece, (2) laser diode and collimator, (3) parts to hold polarizing film, and (4) photodiodes. (b) Photo of the probe in hand.

replicated using the proper combination and concentration of pigments. The reduced scattering coefficient $\mu_{\mathrm{s}}^{\prime}$ and absorption coefficient $\mu_{\mathrm{a}}$ of the phantoms were determined using a red diode laser (B\&WTek Flex, $5 \mathrm{~mW}, \lambda=663 \mathrm{~nm}$ ) according to the integration sphere approach, ${ }^{27}$ while a ballistic photon detection technique ${ }^{22}$ and the inverse adding-doubling software from the Oregon Medical Laser Center were applied to calculate the anisotropy $g$ of the phantoms. ${ }^{28}$ Table 1 shows the properties of the four rough color phantoms P1 through P4 with distinct optical properties. Phantoms P1 and P4 had different $\mu_{\mathrm{a}}$ values $\left(0.284\right.$ and $\left.0.094 \mathrm{~mm}^{-1}\right)$, although their $\mu_{\mathrm{s}}^{\prime}$ values $(0.507$ and $0.549 \mathrm{~mm}^{-1}$ ) are similar. There is a good separation in $\mu_{\mathrm{s}}^{\prime}$ for P2 and P4 (1.285 and $\left.0.549 \mathrm{~cm}^{-1}\right)$, although their $\mu_{\mathrm{a}}$ are indistinguishable $\left(0.085 \pm 0.016\right.$ and $\left.0.094 \pm 0.018 \mathrm{~cm}^{-1}\right)$. The $\mu_{\mathrm{s}}^{\prime}$ of P3, $0.780 \mathrm{~cm}^{-1}$, was in between the values for P2 and P4. On the other hand, the anisotropy $g$ values for all four phantoms were similar. Table 1 also reports published optical properties for normal skin, the benign lesion SK, BCC, SCC, and MM. ${ }^{29,30}$ The phantoms' absorption is in line with the published values; however, scattering tends to be lower than the published values.

\section{Results}

\subsection{Skin Phantom Measurements}

For each of the roughness zones on the skin phantoms, the Stokes polarimetry probe measured the DOP of backscattered light for both linear $\left(\mathrm{DOP}_{\mathrm{L}}\right)$ and circular $\left(\mathrm{DOP}_{\mathrm{C}}\right)$ input polarizations. These measurements of DOP versus roughness are reported in Fig. 2, including trend lines. Consistent with previous studies of depolarization and roughness, ${ }^{17}$ increasing roughness has a stronger depolarizing effect. 
Table 1 Bulk optical properties of phantoms and skin lesions at $\lambda=663 \mathrm{~nm}$.

\begin{tabular}{|c|c|c|c|c|}
\hline & $\begin{array}{c}\text { Absorption } \\
\text { coefficient } \mu_{\mathrm{a}} \mathrm{mm}^{-1} \\
\text { (stderr) }\end{array}$ & $\begin{array}{l}\text { Reduced scattering } \\
\text { coefficient } \mu_{\mathrm{s}}^{\prime} \mathrm{mm}^{-1} \\
\text { (stderr) }\end{array}$ & $\begin{array}{c}\text { Scattering coefficient } \\
\mu_{\mathrm{s}} \mathrm{mm}^{-1} \text { (stderr) }\end{array}$ & $\begin{array}{c}\text { Anisotropy } g \text { a.u. } \\
\text { (stderr) }\end{array}$ \\
\hline Phantom P1 & $0.28(0.02)$ & $0.50(0.01)$ & $2.51(0.22)$ & $0.82(0.07)$ \\
\hline Phantom P2 & $0.09(0.02)$ & $1.29(0.05)$ & $4.12(0.83)$ & $0.69(0.14)$ \\
\hline Phantom P3 & $0.17(0.02)$ & $0.78(0.14)$ & $4.23(0.70)$ & $0.82(0.20)$ \\
\hline Phantom P4 & $0.09(0.02)$ & $0.55(0.02)$ & $2.29(0.50)$ & $0.76(0.16)$ \\
\hline Normal skin (in vivo) ${ }^{29}$ & 0.03 & 1.13 & & \\
\hline $\mathrm{BCC}(\text { in vivo })^{29}$ & 0.05 & 1.90 & & \\
\hline $\mathrm{BCC}(\text { in vitro })^{30}$ & 0.14 & 2.59 & & \\
\hline SCC (in vivo) ${ }^{29}$ & 0.06 & 1.08 & & \\
\hline SCC (in vitro) $)^{30}$ & 0.11 & 1.71 & & \\
\hline $\mathrm{MM}(\text { in vivo })^{29}$ & 0.07 & 1.91 & & \\
\hline SK $(\text { in vivo })^{29}$ & 0.05 & 1.61 & & \\
\hline
\end{tabular}
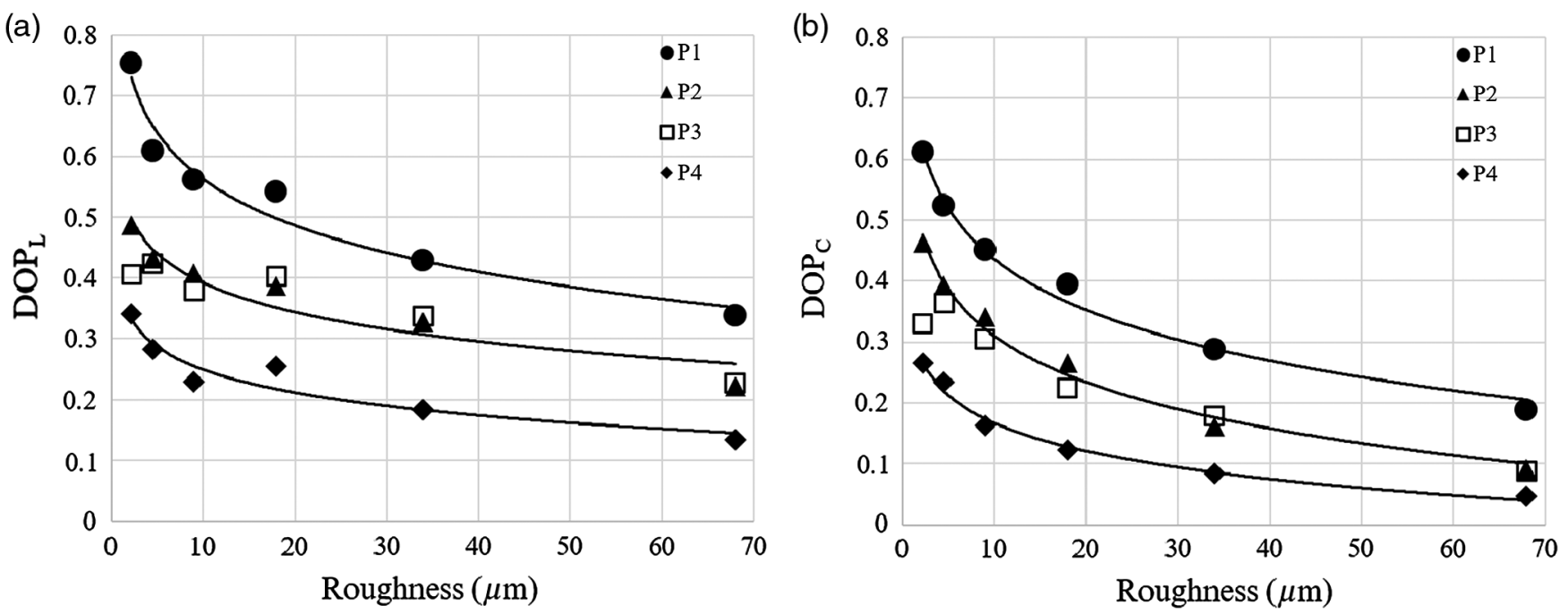

Fig. 2 DOP versus phantom roughness with trend lines for (a) linear and (b) circular input polarizations. Trend lines shown for P1, P2, and P4.

Figure 2 shows the relationship between DOP and surface roughness, the trend of which can be modeled with a log function DOP $=m \cdot \log (R)+C$. The parameters $m$ and $C$ are constants determined by optical properties and $R$ is the surface roughness. The parameter $m$ is indicative of the trendline's curvature, with higher absolute values indicating a greater slope that represents higher sensitivity to roughness. The parameter $C$ indicates the relative height of the curve and relates to the overall DOP. Comparing the trend lines between the $\mathrm{DOP}_{\mathrm{L}}$ and $\mathrm{DOP}_{\mathrm{C}}$ results, the average $m$ of the trendlines is -0.078 for $\mathrm{DOP}_{\mathrm{L}}$ and -0.098 for $\mathrm{DOP}_{\mathrm{C}}$, suggesting that $\mathrm{DOP}_{\mathrm{C}}$ is more sensitive to roughness than $\mathrm{DOP}_{\mathrm{L}}$. In addition, circular polarized light is more strongly depolarized by the phantoms than linear polarized light at each roughness as indicated by the mean $C$ of the trend lines, 0.58 for $\mathrm{DOP}_{\mathrm{L}}$ and 0.53 for $\mathrm{DOP}_{\mathrm{C}}$.
Also by inspecting these trendlines, the curves followed by each phantom appear to differentiate by the parameter $C$. This difference between phantoms is likely due to their different optical properties independent of roughness. This finding reflects polarimetric sensitivity to volume scattering and absorption, which are therefore important to measure as these are the properties that would differ more between cancerous and benign lesions than surface roughness.

\subsection{Clinical Evaluation of Skin Lesions In Vivo}

This pilot clinical trial was performed with volunteer patients at the Vancouver General Hospital Skin Care Centre in Vancouver, Canada. The study was approved the University of British Columbia research ethical board, H06-70281. We measured 
69 lesions in vivo across 47 patients. Lesions were identified by a dermatologist, and if suspected to be cancerous, their diagnoses were confirmed through histological analysis following a biopsy. Lesions were grouped into seven categories: MM, benign nevus (BN), BCC, seborrheic keratosis (SK), SCC, actinic keratosis (AK), and other benign lesions (OB). The nevi were atypical on observation and biopsied on suspicion of cancer. The OB category included dermatofibroma, lentigo, neurofibroma, cherry angioma, and scar tissue. Each lesion was measured, and the $\mathrm{DOP}_{\mathrm{L}}$ and $\mathrm{DOP}_{\mathrm{C}}$ were calculated for each.

The five lesions in the OB category were omitted from analysis, as it contains a variety of individual lesion types that should not be analyzed together. In addition, lesions were also excluded from averaging due to device malfunction errors such as the mislabeling of input polarization, or probe misalignment leading to nonuniformity between the four photodiodes. For linear polarized input light, $1 \mathrm{BCC}$ was excluded for this reason. For circular polarized input light, five lesions were excluded: $1 \mathrm{BCC}, 1$ SK, 2 SCC, and 1 AK. Finally, the probe was constructed take measurements from a normal direction to the lesion surface, and measurements taken from body sites that were not relatively flat, such as the ear and nose, were also excluded. Four data points were excluded for both input polarizations for this reason: $1 \mathrm{BN}$, $2 \mathrm{BCC}$, and $1 \mathrm{SCC}$. This protocol filtered the results down to a total of 59 lesion measurements with linear polarized input light and 55 lesion measurements with circular polarized input light. The final counts are shown in Table 2, which also summarizes the mean and standard error of the collected measurements of all tested lesion types. Figure 3 visualizes this data and to demonstrate the effect of roughness on DOP in these measurements the lesions are sorted in order of mean roughness from smoothest to roughest. The roughness values were determined through a study of lesion roughness using the depolarization ratio. ${ }^{31}$ The report on the thorough analysis of mean surface roughness and skin lesion types is under preparation. For this paper, we used only the relative ranks of lesion roughness, such as MM tending to have the smoothest surface and AK tending to have the roughest among the studied lesion types. These rankings agree with the common notion of roughness among most dermatologists.

The most striking observation in these results is the greater mean $\mathrm{DOP}_{\mathrm{L}}$ and $\mathrm{DOP}_{\mathrm{C}}$ for melanoma over any other lesions.

Table 2 Clinical trial results summary: each lesion type, the number of cases measured, and mean DOP for both input polarizations.

\begin{tabular}{lcccc} 
Lesion & $\begin{array}{c}\text { Count } \\
\text { (linear } \\
\text { Input) }\end{array}$ & $\begin{array}{c}\text { Count } \\
\text { (circular } \\
\text { input) }\end{array}$ & $\begin{array}{c}\text { Mean DOP } \\
\text { a.u. (stderr) }\end{array}$ & $\begin{array}{c}\text { Mean DOP. (stderr) } \\
\text { a.u }\end{array}$ \\
\hline MM & 5 & 5 & $0.46(0.09)$ & $0.45(0.10)$ \\
BN & 5 & 5 & $0.31(0.06)$ & $0.18(0.03)$ \\
BCC & 15 & 15 & $0.28(0.03)$ & $0.19(0.02)$ \\
SK & 16 & 15 & $0.28(0.02)$ & $0.18(0.02)$ \\
SCC & 9 & 7 & $0.26(0.03)$ & $0.19(0.03)$ \\
AK & 9 & 8 & $0.26(0.02)$ & $0.21(0.03)$ \\
All \\
nonmelanoma
\end{tabular}

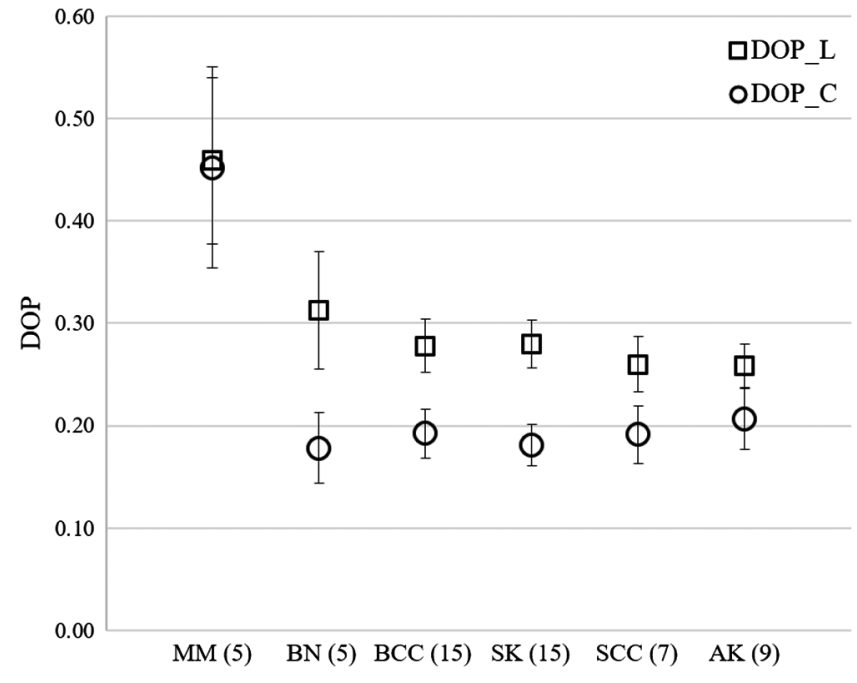

Fig. 3 Mean DOP measurements for skin lesion types. The order of lesions types is based on the ranking of skin lesion roughness.

Noting the standard errors, the measured mean $\mathrm{DOP}_{\mathrm{L}}$ for $\mathrm{MM}(0.46 \pm 0.09)$ and $\mathrm{BN}(0.31 \pm 0.06)$ are distinguishable by a small margin, while the measured mean $\mathrm{DOP}_{\mathrm{C}}$ for $\mathrm{MM}$ $(0.45 \pm 0.10)$ stands clearly separate from $\mathrm{BN}(0.18 \pm 0.03)$. For both input polarizations, MM is distinguishable from the mean DOP values of all other lesions combined. In clinical practice, melanoma is identified as smoother than other skin lesions, which may be the predominant reason for this difference. Another relevant observation is the pattern across all lesions that the $\mathrm{DOP}_{\mathrm{C}}$ is lower than the $\mathrm{DOP}_{\mathrm{L}}$, and the difference between the two may communicate a relevant measurement. These findings are the basis for further investigations into the effect of skin lesions on polarized light.

\section{Limitations and Conclusions}

\subsection{Trial Limitations}

The Stokes polarimetry probe was designed with compact size and cost-effectiveness in mind. While testing on skin phantoms in a controlled setting yielded reasonable results, clinical testing revealed that a more robust polarimetric measurement system will be required. For example, parallel alignment of the detector plane to the target surface is especially crucial to ensure symmetry among the four free-space detectors, and this is disrupted by probe misalignment due to any possible angled orientation with respect to the lesion. Designing a more rigorous alignment method in the probe or including measurement checks for alignment errors will be necessary. Reducing the impact of error caused by field nonuniformity could potentially be improved using a different four-filter scheme. As proposed by Hsu et al., ${ }^{32}$ taking four measurements in equidistant polarization states using elliptical polarizers will better balance against errors.

The skin phantoms measured in this study replicate the roughness and absorption properties of skin lesions and were found to have consistently lower scattering coefficients. This was intentional, as surface roughness has the predominant effect on DOP. Creating phantoms to additionally replicate the scattering properties of skin is a nontrivial task and may be the subject of future study. 
The clinical trial presented here was a proof of principle field test of the probe and the potential utility of DOP measurements. While the difference in DOP for melanoma is notable, a low number of melanoma and nevus lesions were measured, and this is too small a sample size to perform a valid statistical significance test. More extensive clinical testing in the future, with a greater number of lesions and an updated device, will be required to validate a clinical claim.

\subsection{Conclusions}

The DOP is shown to be a potentially useful diagnostic property to identify melanoma among other types of skin lesions. A oneshot Stokes polarimetry probe was used to analyze the polarimetric properties of skin lesions in vivo by measuring the Stokes parameters of backscattered laser light in both linear and circular initial polarization.

Through trials on rough skin phantoms, it was demonstrated that there is a correlation between backscattered DOP and skin roughness. This correlation exists for both linear and circular input polarization and was found to be a more sensitive metric using circular polarized input. In addition, the optical properties of the skin phantom's volume such as absorption and scattering allowed for the differentiation of skin phantoms through DOP independent of surface roughness. In a pilot clinical trial where a variety of cancerous and benign skin lesions were tested in vivo, it was found that the DOP for melanoma lesions was measured to be greater than that of other lesions. This separation is greater for circular polarized input light, and it is likely that circular polarized light's greater sensitivity to surface roughness contributes to this result. In addition, skin lesions appear to have a stronger depolarizing effect on circular polarized light than linear polarized light, as demonstrated by a lower mean DOP for circular polarized input light across all lesion types evaluated.

\section{Disclosures}

Louie D.C., Tchvialeva L., Lui H., and Lee T.K. report patents, which are owned by British Columbia Cancer Research Centre and licensed to commercial entities, that are related to the technology and analysis methods described in this study. This research was conducted without the participation, knowledge, or financial support of these entities.

\section{Acknowledgments}

We thank all our study participants, and the clinical staff at the Vancouver General Hospital Skin Care Centre for your time and other contributions to this project. This work was supported in part by grants from the Canadian Dermatology Foundation, the VGH and UBC Hospital Foundation, and NSERC (2017-04932).

\section{References}

1. Z. Apalla et al., "Skin cancer: epidemiology, disease burden, pathophysiology, diagnosis, and therapeutic approaches," Dermatol. Ther. 7(1), 5-19 (2017).

2. A. Fahradyan et al., "Updates on the management of non-melanoma skin cancer," Healthcare 5, 82 (2017).

3. S. Leachman et al., "Methods of melanoma detection," Melanoma 167, 51-105 (2015).

4. Y. Pan et al., "Polarized and nonpolarized dermoscopy: the explanation for the observed differences," Arch. Dermatol. 144(6), 828-829 (2008).
5. C. Okoro and K. Toussaint, "Second-harmonic patterned polarizationanalyzed reflection confocal microscopy of collagen," J. Biomed. Opt. 22(8), 086007 (2017).

6. M. J. Ju et al., "Advanced multi-contrast Jones matrix optical coherence tomography for Doppler and polarization sensitive imaging," Opt. Express 21(16), 19412-19436 (2013).

7. N. Ghosh and A. I. Vitkin, "Tissue polarimetry: concepts, challenged, applications, and outlook," J. Biomed. Opt. 16(11), 110801 (2011).

8. S. Alali and I. A. Vitkin, "Polarized light imaging in biomedicine: emerging Mueller matrix methodologies for bulk tissue assessment," J. Biomed. Opt. 20(6), 061104 (2015).

9. S. Alali, A. Gribble, and A. I. Vitkin, "Rapid wide-field Mueller matrix polarimetry imaging based on four photoelastic modulators with no moving parts," Opt. Lett. 41(5), 1038-1041 (2016).

10. J. Qi, C. He, and D. S. Elson, "Real time complete Stokes polarimetric imager based on a linear polarizer array camera for tissue polarimetric imaging," Biomed. Opt. Express 8(11), 4933-4946 (2017).

11. P. Ghassemi and M. H. Miranbaygi, "Towards skin polarization characterization using polarimetric technique," J. Zhejiang Univ. 10(8), 602-608 (2009).

12. B. Kunnen et al., "Application of circularly polarized light for non-invasive diagnosis of cancerous tissues and turbid tissue-like scattering media," J. Biophotonics 8(4), 317-323 (2015).

13. P. Ghassemi et al., "Out-of-plane Stokes imaging polarimeter for early skin cancer diagnosis," J. Biomed. Opt. 17(7), 076014 (2012).

14. J. Chue-Sang et al., "Use of Mueller matrix polarimetry and optical coherence tomography in the characterization of cervical collagen anisotropy," J. Biomed. Opt. 22(8), 086010 (2017).

15. S. Kumar et al., "Comparative study of differential matrix and extended polar decomposition formalisms for polarimetric characterization of complex tissue-like turbid media," J. Biomed. Opt. 17(10), 105006 (2012).

16. A. Vitkin, N. Ghosh, and A. de Martino, "Tissue polarimetry," in Photonics: Scientific Foundations, Technology and Applications, D. L. Andrews, Ed., Vol. IV, pp. 239-321, John Wiley and Sons, Inc., Hoboken, New Jersey (2015).

17. L. Tchvialeva et al., "Polarization speckle imaging as a potential technique for in vivo skin cancer detection," J. Biomed. Opt. 18(6), 061211 (2013).

18. D. C. Louie et al., "Stokes polarimetry probe for skin lesion evaluation: preliminary results," Proc. SPIE 10467, 104670X (2018).

19. E. Collett, "Measurement of the four Stokes polarization parameters with a single circular polarizer," Opt. Commun. 52(2), 77-80 (1984).

20. D. C. Louie, L. Tchvialeva, and T. K. Lee, "Proof of principle of a Stokes polarimetry probe for skin lesion evaluation," in IEEE EMBS Int. Student Conf., Ottawa (2016).

21. I. Markhvida et al., "Influence of geometry on polychromatic speckle contrast," J. Opt. Soc. Am. A 24(1), 93-97 (2007).

22. D. Diao et al., "Durable rough skin phantom for optical modelling," Phys. Med. Biol. 59(2), 485-492 (2014).

23. C. Hof, "Application of wavelet- and wavelet-packet-transform to human skin data," Proc. SPIE 4474, 9-19 (2001).

24. L. Li et al., "Age-related changes in skin topography and microcirculation," Arch. Dermatol. Res. 297(9), 412-416 (2006).

25. G. G. Hillebrand et al., "Quantitative evaluation of skin condition in an epidemiological survey of females living in northern versus southern Japan,” J. Dermatol. Sci. 27(Suppl. 1), 42-52 (2001).

26. B. Connemann et al., "Sources of unwanted variability in measurement and description of skin surface topography," Skin Res. Technol. 2, 40-48 (1996).

27. S. A. Prahl, "Light transport in tissue," $\mathrm{PhD}$ dissertation, University of Texas at Austin (1988).

28. S. A. Prahl, "Inverse adding-doubling," 2007, http://omlc.org/software/ $\mathrm{iad} /$ index.html

29. A. Garcia-Uribe et al., "In vivo diagnosis of melanoma and nonmelanoma skin cancer using oblique incidence diffuse reflectance spectrometry," Cancer Res. 72(11), 2738-2745 (2012).

30. E. Salomatina et al., "Optical properties of normal and cancerous human skin in the visible and near-infrared spectral range," J. Biomed. Opt. 11(6), 064026 (2006).

31. T. K. Lee et al., "Applications of polarization speckle in skin cancer detection and monitoring," Proc. SPIE 10612, 106121B (2017). 
32. W. Hsu et al., "Full-Stokes imaging polarimeter using an array of elliptical polarizer," Opt. Express 22(3), 3063-3074 (2014).

Daniel C. Louie is a PhD candidate in the School of Biomedical Engineering at the University of British Columbia. His proposed dissertation is on the measurement of polarization speckle for skin assessment and cancer detection. His research interests include the adaptation of optical technology for widely available public health applications. He works with British Columbia Cancer Control Research and the Photomedicine Institute at the UBC Department of Dermatology and Skin Science.

Jamie Phillips is a resident in the Department of Dermatology and Skin Science at the University of British Columbia. Prior to Medical School, he studied biology and physics where he took a keen interest in skin science and biomedical optics. During medical school, he became involved with polarization speckle research for skin cancer detection. $\mathrm{He}$ is particularly interested in translational research and the intersection of basic science with clinical medicine.

Lioudmila Tchvialeva is a lecturer with the physics department at the University of British Columbia (UBC). Her research interests include the propagation of coherent polarized light in turbid tissue-like scattering media, tissue polarimetry, and applications of coherent light for skin diseases. Currently, she holds a researcher associate position in the Photomedicine Institute at the UBC Department of Dermatology and Skin Science.

Sunil Kalia is an associate professor in the Department of Dermatology and Skin Science at the University of British Columbia.
His clinical and research interests include photodermatology, phototherapy, and epidemiology and health outcomes of dermatologic conditions. He also works with other investigators in the Photomedicine Institute to study optical devices for diagnosing skin conditions.

Harvey Lui is a professor of dermatology and skin science at the University of British Columbia. After his medical and residency training, he completed an advanced fellowship in lasers, photomedicine, and photodynamic therapy at Massachusetts General Hospital. His expertise also includes biomedical optics, pigmentary disorders, and medical education. He has published over 180 peerreviewed papers and has 7 patents. He is the current president of the International League of Dermatological Societies.

Wei Wang is an assistant professor at Heriot-Watt University. His research interests are around statistical optics, optical information processing, imaging science, and their applications to optical metrology and sensing. He has published over 60 papers and was awarded the best science paper award of the Japan Society for Applied Physics in 2007 (the highest research award of Optical Society of Japan). He is a member of the SPIE, OSA, and JSAP.

Tim K. Lee is an associate professor at the University of British Columbia and a senior scientist at BC Cancer. His research interests include skin cancer detection based on optical and artificial intelligence approaches. His developed techniques include polarization speckle for melanoma detection, laser speckle for skin surface roughness quantification, and deep learning algorithms for extracting and analyzing diagnostic features from dermoscopic and wide-area skin images. He is a senior member of SPIE. 